\title{
Exploiting Modularity, Hierarchy, and Repetition in Variable-Length Problems
}

\author{
Edwin D. de Jong ${ }^{\text {a }} \quad$ Dirk Thierens ${ }^{a}$ \\ ${ }^{a}$ Decision Support Systems Group, Universiteit Utrecht. \\ E-mail: \{dejong, dirk.thierens\}@cs.uu.nl
}

\section{Keywords: Modularity, hierarchy, repetition, SEQ, HSEQ problem}

\section{Full version:}

De Jong, E.D. and Thierens, D. (2004). Exploiting Modularity, Hierarchy, and Repetition in Variable-Length Problems. In: Proceedings of the Genetic and Evolutionary Computation Conference, GECCO-04, pp. 1030-1041.

URL: http://www.cs.uu.nl/ dejong

Currently, evolutionary computation can reliably address problems for which the order of the dependencies between the $n$ variables is limited to a small number $k$, where two variables are called dependent if the fitness contribution of one variable depends on the setting of the other variable and the order of the dependencies is the largest number of interdependent variables. Apart from these problems, there are certain specific problems with higher-order dependencies that can also be addressed. Indeed, hierarchical problems with dependencies up to order $k=n$ can still be solvable in a scalable manner. Examples of hierarchical problems so far include H-IFF, H-TRAP, and H-XOR, which can be addressed by methods such as SEAM [3] and H-BOA [2].

The class of hierarchical fixed-length problems is of interest because it is the most complex problem class that may still be efficiently addressed by currently known evolutionary algorithms. The class of feasible problems may be further extended however if variable-length hierarchical problems can be addressed. A potential in this direction is demonstrated by the DevRep algorithm [1]; this method was reported to address a 1024-bit version of the HXOR problem. While HXOR indeed features modularity, hierarchy, and repetition, this result does not demonstrate that the DevRep algorithm can exploit these problem features in isolation.

Our aim will be to explore whether modularity, hierarchy, and repetition can be identified in isolation. Existing test problems contain a combination of these features. Therefore, we introduce new test problems that enable the study of these problem features in isolation. The Sequence problem (SEQ) features modularity, but no hierarchy or repetition. The Hierarchical Sequence problem (HSEQ) features hierarchy but no repetition. To study repetition, we employ the OneMax problem.

We develop a variable length algorithm for module formation. The operation of the algorithm on the SEQ, HSEQ, and OneMax problems is studied in experiments. 
Control experiments are performed to analyze the necessity of different features of the algorithm.
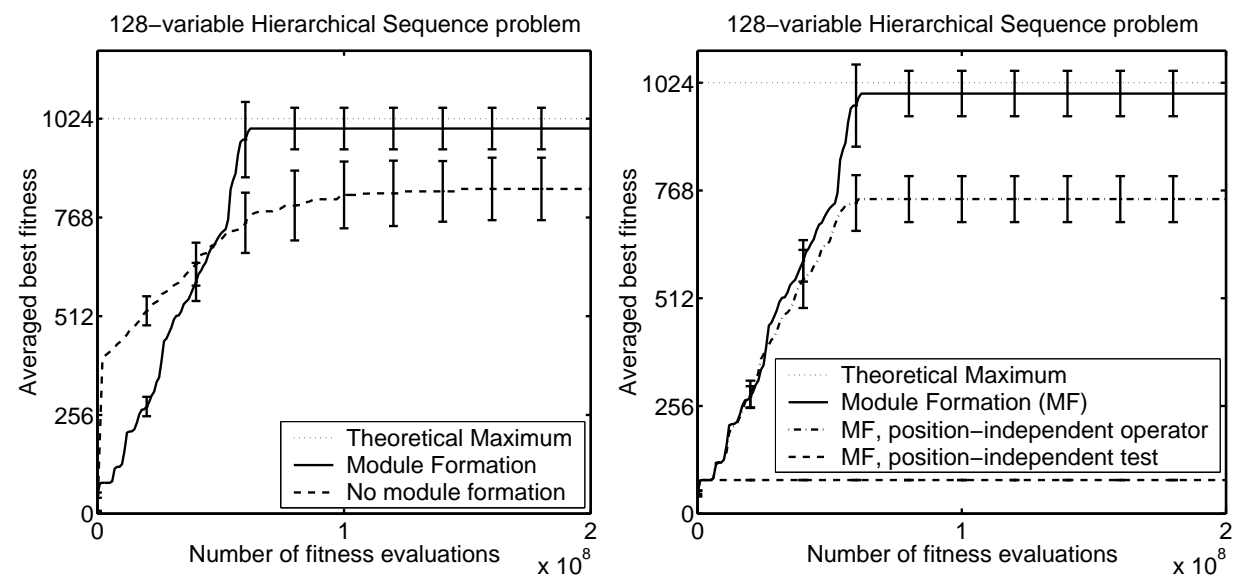

Figure 1: Performance on the 128-variable HSEQ problem for various methods.

It is demonstrated that the modules formed by the method correspond to the modules present in the problems, and the method can thus be said to detect modularity, hierarchy, and repetition to a substantial degree. For the HSEQ and OneMax problems, a significant performance gain was achieved as a result of module formation. While translocation is seen to be useful in the presence of repetition and no insurmountable obstacle in the hierarchical HSEQ problem, a position-specific module-acceptance test was found crucial in the latter problem. These findings suggest that successful exploitation of both hierarchy and repetition will require both position-specific module testing and position-independent module use.

\section{References}

[1] Edwin D. De Jong. Representation development from Pareto-coevolution. In E. Cantú-Paz et al., editor, Proceedings of the Genetic and Evolutionary Computation Conference, GECCO-03, pages 262-273, Berlin, 2003. Springer.

[2] Martin Pelikan and David E. Goldberg. Escaping hierarchical traps with competent genetic algorithms. In L. Spector et al., editor, Proceedings of the Genetic and Evolutionary Computation Conference, GECCO-01, pages 511-518. Morgan Kaufmann, 2001.

[3] Richard A. Watson and Jordan B. Pollack. A computational model of symbiotic composition in evolutionary transitions. Biosystems, 69(2-3):187-209, May 2003. Special Issue on Evolvability, ed. Nehaniv. 